Monatsschrift f. Geburtshülfe u. Gynäkologie 1929;82:I-IV

\title{
Contents, Vol. 82, 1929
}

Inhaltsverzeichnis.

Originalarbeiten. S $\quad$ ite

Adler, Karl und Ernst Böltink, ßeitrag zur Wirkung der Vitamine

auf die Funktion der Ovarien im Tierexperiinent

19

Böltink, Ernst, siehe Adler, Karl.

Brandis, E., Über Nabelschnurvorfall208

Brühl, R., Kapselruptur eines intramuralen Myoms 287

Fraenkel, L., Abdominale und vaginale Operationsmethoden in der

Geburtshilfe und Gynäkologie 79

Freund, Ludwig, Embryonalentwicklung und Tumorbereitschaft. Eine

Studie zur Geschwulstlehre von Cohnheim 276

Geller, Chr., Über die Dauererfolge der operativen Behandluog von

Adnexentzündungen 296

- $\quad$ Naehwort zu dieser Arbeit 450

Gutman, Martin, Die bakterizide Kraft im Blute des Neugeborenen 93 Halban, J. und Milan Z.

Spitzer, Über das gesteigerte Wachstum

der Nägel in der Schwangerschaft 25

Heck seller, S., Der Kaufftnannsche Diureseversuch und seine Be-

deutung für die operative Tätigkeit des Gynäkologen

Joseph, S. uñd Karl Mayer, Entzündungsbestrahluлgen entzündlicher

Adnexerkrankungen 438

Kaiser, Kurt, Spätdehiszenzen alter Bauchnarben

322

- $\quad$ Geheilte Quecksilbervergif'tung $\quad 325$

Kleiner, B., Ein seltener Fall von Doppelbildung einer Frucht - Duplicitas anterior (Dicephalus)

Koerner, Johannes, Überdrehung des hochschwangeren Uterus . . 259

Lahm, W., Uterusblutungen in der Menopause

- $\quad$ Zur Pathologie der basalen Schicht der Uterusschleimhaut

Lwow, N. A., Uber die diagnostisehe Bedeutung der Reaktion Bordet-

Gengou in der Klinik der weiblichen Gonorrhöe 305

Martin, Ed., „Unden” bei der Aufzucht von Frühgeburten 66

Mayer, Karl, siehe Joseph, S.

MussatO $\Lambda$ V, N. A., Die intrauterinen Eingießungen als gynäkologische

Heilmethode. Vorläufige Mitteilung · 204

Nahmmaeher, H., Zur Krebsfrage und die Heilungsresultate beim gynäkologischen Karzinom. (Resultate der Universitäts-Frauenklinik Jena 1919-1925.) 291

Naujoks, H, Zur Begriffsbestimmung in der gynäkologischen Röntgenologie, beso $\pi$ ders im Hinblick auf die Frage der Nachkommen- 
schädigung 429

Pfleiderer, A., Über Gebärmuttermißbildungen 401

Puppel, Ernst, Osteogenesis imperfecta 269

Rummel, Hans, Zur Kenntnis der mütterlichen traumatischen Ent-

bindungslähmung $\quad 31$

Russin, Jakob, Die spontane Zerreißung der velamentös inserierten

Nabelschnur 264

S c h e n k , F., Spätbefunde an der Hypophyse von spätkastrierten Ratten 424 Sdrawomyslow,

W. J., Die künstliche Scheidenbildung nach der

Methode von Prof D. D. Popoff und ihre Dauererfolge 182

Sellheim,Hugo, Erleichterung der Myomoperation von unten und oben 70 Spitzer, Milan Z, siehe Halban, J.

jy Inhaltsverzeichnis.

Seit $\theta$ Steinhardt, Bianca, Sekundäres Uterussarkom nach langer Latenzzeit 168 Vögel, J., Das Geburtstrauma und seine Bedeutung in der Geburtshilfe 40 Wagner, G. A., Hypophyse und weibliches Genitale. Hypophyse und

Schwangerschaft 1

Wolf, Herbert, Operative Kastration nach erfolgloser Eöntgen-

kastration $\quad 312$

Robert Asch $\dagger 156$

Friedrich Ahlfeld $\dagger \quad 473$

Sammelberichte.

Die Wasserstoffionenkonzentration und ihre Bedeutung für die Gynäko-

logie. Von Dr. Paul W i r z - Frankfurt am Main 103

Berichte fiber ausländische Literatur.

Die französische geburtshilflich-gygäkologische Literatur (1928). Von Dr.

H. Vignes-Paris 112

Übersicht der geburtshilf lichen und gynäkologischen Literatur von U.S.S.E.

im Jahre 1927. Von E. M. Schwarzmann-Moskau 125

Die italienische geburtshilflich-gynäkologische Literatur (1928. II. Semester).

Von Privatdozent Dr. Sebastiano di Francesco-Mailand . . . 217 Schwedische geburtshilflich-

gynäkologische Literatur des Jahres 1928.

Von Seved Bergendal-Luad 451

Verei $\pi$ s- und Líteraturbeilage. Original-Sitzungsberichte aus geburtshilflich-gynäkologischen

Gesellschaften:

Gynäkologische Gesellschaft zu Breslau. Sitzung vom 15. I. 1928 . . 145

Sitzungen vom 19. III. und 23. IV. 1929361

G-esellschaft für Geburtshilfe und Gynäkologie in Berlin.

Sitzungen vom 11. 1. und 25. I. 1929236

Sitzungen vom 8. II. und 8. III. 1929342

Sitzungen vom 22. II. und 26. IV. 1929457

Mittelrheinische Gesellschaft für Geburtshilfe und Gynäkologie. 78. Sitzung

vom 17. II. 1929 in Frankfurt a. M 242

Südostdeutsche Gesellschaft für Geburtshilfe und Gynäkologie. V. Tagung

am 20. und 21. X. 1929 in Eeichenberg (C.S.E.) 370

Geburtshilflich-gynäkologische GesellschaftinWien. Sitzung vom 19.Ill.1929 369 
Sitzungen vom 14. V. und 11. VI. $1929 \quad 465$

Der 21. Kongreß der Deutschen Gesellschaft für Gynäkologie zu Leipzig.

Von Ed. Martin-Elberfeld 329

- Sitzungsbericht. Von G. H. Schneider-Brandenburg

233,331

Aus dem 20. Deutschen Eöntgenkongreß in Wien (19. bis 23. IV. 1929).

Von G. H. Schneider-Brandenburg 395

Aus dem 53. Deutschen Chirurgenkongreß 1929 zu Berlin, $\Lambda^{\wedge}$ on G. $\mathrm{H}$.

S chn eider-Brandenburg 397

Buchbesprechangen 147, 257, 399, 468

Personalien und Tagesnachrichten 144, 400, 474

Druckfehlerberichtigu $\prod g$ zu den Arbeiten „Dienst” in dieser Monatsschrift, Bd. 77 und 79258 